MONITORING THE FINISH-LINE: INTEGRATED GLOBAL CRITERIA AND PROCESSES FOR VALIDATION OF EMTCT OF SYPHILIS AND HIV

doi:10.1136/sextrans-2013-051184.0068

'L Newman, ${ }^{2} \mathrm{C}$ Hayashi. 'Department of Reproductive Health \& Research, WHO, Geneva, Switzerland; '2Department of HIVIAIDS, WHO, Gemeva, Switzerland

Background The global community is committed to elimination of mother-to-child transmission (EMTCT) of HIV and syphilis as public health problems. International and regional goals have been set, and countries are scaling up programmes towards EMTCT. Regional initiatives in the Americas, Asia-Pacific, and Africa have approached control of MTCT of HIV and syphilis as an integrated process. Prior to this work, there were no internationally standardised processes and criteria to validate EMTCT of HIV or syphilis.

Methods As the global community prepares to assess progress towards global health goals in 2015 and beyond, standardised processes and criteria are needed to assess and validate EMTCT of HIV and syphilis across widely varying epidemiologic and programmatic contexts. This presentation will review the process for development of minimum global processes and criteria, provide a description of global validation targets and indicators, explain validation procedures, including maintenance of validation status, and describe next steps at the global, regional, and national levels.

Results These guidelines are intended for use by national, regional, and global validation committees; national AIDS, sexually transmitted infection, maternal, and child health programme managers; monitoring and evaluation (M\&E) officers; policy-makers; and international partners. Currently there are at least 4 regions moving forward with establishment of regional processes for validation, and several countries have expressed interest in applying for validation of EMTCT of HIV and/or syphilis.

Conclusions As experience is gained through establishment of global and regional validation processes, additional guidance and tools will be developed to complement these initial minimum global standards. Although it is recognised that not all countries are able to meet these criteria at this time, these criteria can also serve as aspirational targets for countries for 2015 and beyond and an opportunity to improve programmes and monitoring systems, reduce disparities within countries, and highlight models of success.

\section{S14.5 PILOTING METHODS FOR VALIDATION OF ELIMINATION OF MTCT OF HIV AND SYPHILIS IN ST. LUCIA AND CHILE}

doi:10.1136/sextrans-2013-051184.0069

${ }^{1} \mathbf{K}$ W Hoover, ${ }^{2} \mathrm{~S}$ Caffe, ${ }^{2} \mathrm{M}$ Alonso, 'M Kamb. ' $\mathrm{C} D \mathrm{C}$, Atlanta, GA, United States, ${ }^{2}$ Pan American Health Organization, Washington, DC, United States

Introduction In 2010, the Pan American Health Organization (PAHO) member countries approved the Strategy and Plan of Action for Elimination of Mother-to-Child Transmission (MTCT) of HIV and Congenital Syphilis, with the goal of elimination by 2015. Elimination targets are MTCT of syphilis of $\leq 0.5$ cases per 1,000 live births; MTCT of HIV of $\leq 0.3$ cases per 1,000 live births; and transmission of HIV from $\mathrm{HV}$-infected mothers to their infants of $\leq 2 \%$. PAHO has supported Latin American and Caribbean countries to achieve these goals, by developing several technical resources, a system for reporting indicators of elimination, and methodology for validating elimination.

Methods In 2012, we piloted validation methodology in St. Lucia and Chile, countries with robust elimination programmes. Teams of external experts travelled to each country to assess the national surveillance system and verify reported data; the national laboratory system; and programmatic aspects important for achieving and sustaining elimination. The assessment included review of databases and reports, triangulation and re-calculation of data, mapping of health services delivery, interviews with national and regional stakeholders, and visits to service delivery sites.

Results The validation methodology was able to identify national strengths and challenges in surveillance, health services, laboratory, and programme. It also was able to demonstrate sub-national variances and inequities in achieving and maintaining elimination targets, and barriers to health services access in underserved populations. Data verification and triangulation led to re-calculation of some indicators, but others could not be calculated due to data gaps.

Conclusion The validation methodology is useful for assessing progress in elimination of MTCT of HIV and syphilis, but also as a tool for countries to review their programmatic actitivies to identify opportunities for accelerating achievement of elimination. Challenges in recalculation of indicators emphasise the importance of comprehensive information systems that collect data necessary to verify elimination.

\section{S.15 - Sexual networks and contagion (Insights from phylodynamic studies, behaviour diffusion, social media studies)}

\section{S15.1 FREQUENT VIRAL INTRODUCTIONS SUSTAIN LOCAL HIV EPIDEMICS IN RURAL AFRICA}

doi:10.1136/sextrans-2013-051184.0070

${ }^{1}$ M K Grabowski, 'J Lessler, ${ }^{2} \mathrm{~A}$ Redd, ${ }^{3} \mathrm{~J}$ Kagaayi, 1,20 Laeyendecker, ${ }^{3} \mathrm{~F}$ Nalugoda, ${ }^{3,4} \mathrm{D}$ Serwadda, ${ }^{1,3} \mathrm{M}$ Wawer, ${ }^{1,2}$ T C Quinn, ${ }^{1,3} \mathrm{R}$ H Gray. ${ }^{1} \mathrm{~J}$ ohns Hopkins, Baltimore, MD, United States; 'Laboratory of Immunoregulation, Division of Intramural Research, National Institute of Allergy and Infectious Diseases, National Institutes of Health, Bethesda, MD, United States; ${ }^{3}$ Rakai Health Sciences Program, Kalisizo, Uganda, ${ }^{4}$ School of Medicine, Makerere University, Kampala, Uganda

Background It is often assumed that local sexual networks play a dominant role in HIV spread in sub-Saharan Africa. However, the extent to which continued HIV transmission in rural African communities, home to two-thirds of the continent's population, is driven by intra-community sexual networks versus viral introductions from outside of communities remains unknown.

Methods We analysed the spatial dynamics of HIV transmission in rural Rakai District, Uganda using population-based cohort data on 14594 individuals within 46 communities experiencing a generalised HIV epidemic (prevalence $12 \cdot 2 \%$, incidence $\sim 1 \cdot 2$ per 100 person years). We applied spatial clustering statistics, phylogenetic analyses, and probabilistic transmission models to quantify the relative contributions of viral introductions versus local HIV transmission, including household transmission, to HIV incidence.

Results Individuals in households with incident $(\mathrm{n}=189)$ or prevalent $(\mathrm{n}=1597)$ HIV-infected persons were $3 \cdot 2$ (95\% CI: $2 \cdot 7-3 \cdot 7)$ times more likely to be HIV-infected, compared to the population in general, but spatial clustering outside of households was weak and confined to distances $<500 \mathrm{~m}$. There was high viral diversity: only $34 \%$ of infected persons phylogenetically clustered with at least one other participant. One-third of phylogenetic clusters were individuals sharing a household. Of the remaining two-thirds, $71 \%$ crossed community boundaries. We estimate that 39\% (95\% CI: $32-44 \%$ ) of viral transmissions occurred within stable household partnerships and that $40 \%$ (95\% CI: $34-42 \%$ ) of transmissions were from named extra-household sexual contacts, of which $62 \%$ (95\% CI: $55-70 \%$ ) were non-stable partners from outside the community.

Interpretation: External HIV introductions into rural communities are common and account for a substantial proportion of new HIV infections in Rakai, Uganda. Our findings suggest that combination HIV prevention will most effectively control local HIV epidemics when implemented at broad spatial scales, and imply a need to identify key populations serving as sources of introduced infections into general populations. 